\title{
Association of key sources of riboflavin with EGRac status in a representative sample of Irish adults aged 18-90 years
}

\author{
L. Kehoe ${ }^{1}$, J. Walton ${ }^{1,2}$, S.M. Hopkins ${ }^{3}$, B.A. McNulty ${ }^{3}$, A.P. Nugent ${ }^{3,4}$, H. McNulty ${ }^{5}$, \\ M. Ward ${ }^{5}$ and A. Flynn ${ }^{1}$ \\ ${ }^{1}$ School of Food and Nutritional Sciences, University College Cork, Ireland, \\ ${ }^{2}$ Dept. Biological Sciences, Cork Institute of Technology, Ireland, \\ ${ }^{3} U C D$ Institute of Food and Health, University College Dublin, Ireland, \\ ${ }^{4}$ Institute for Global Food Security, Queens University Belfast, N. Ireland and \\ ${ }^{5}$ Nutrition Innovation Centre for Food and Health, Ulster University, Coleraine, N. Ireland
}

Previous analysis has identified that $10-26 \%$ of Irish adults (range across age groups) have low dietary intakes of riboflavin while $61 \%$ have evidence of low/deficient riboflavin biomarker status (erythrocyte glutathione reductase activation coefficient $(\text { EGRac) }>1.3)^{(1)}$. The key dietary sources of riboflavin in the Irish population were identified as milks, 'meat \& meat products' and ready-to-eat breakfast cereals $(\text { RTEBC })^{(1)}$. The objective of this study was to examine the association of consumption of these food groups with EGRac status in Irish adults using data from the National Adult Nutrition Survey (2008-2010) (www.iuna.net). A 4-day semi-weighed food record was used to collect food and beverage intake data from a nationally representative sample of 1500 Irish adults $\left(18-90\right.$ years) ${ }^{(1,2)}$. Blood samples $(n 1126)$ were collected by venepuncture by a trained professional and processed and analysed using stardard operating procedures ${ }^{(1,2)}$. Determination of riboflavin biomarker status was by EGRac, calculated as the ratio of flavin-dependent glutathione reductase activity before and after in vitro reactivation with its prosthetic group $\mathrm{FAD}$, with a cut-off value of $>1.3$ generally used to indicate low/deficient status ${ }^{(3)}$. For the purposes of this analysis; only those participants with both food intake and biochemical data were included ( $n$ 1126). Participants were divided into three intake groups (stratified by age-group and gender); non/low, medium or high consumers of milks, 'meat \& meat products' and RTEBC, respectively; on the basis of their mean daily intake. The prevalence of EGRac $>1.3$ was examined across tertiles of intake of each food group and a chi-square test for inependence was used to determine significant associations $(\mathrm{p}<0.001)$.

The prevalence of low/deficient riboflavin status signficiantly decreased $(\mathrm{p}<0.001)$ across tertile of milk intake: $71 \%$ among those with a non/low intake (equivalent to approx. 0.3 servings/day based on a $200 \mathrm{ml}$ serving) compared to $51 \%$ among those with a high intake (approx. 2.1 servings/day). No significant association was found between tertile of 'meat \& meat products' intake and EGRac status. The prevalence of low/deficient riboflavin status signficiantly decreased $(\mathrm{p}<0.001)$ across tertile of RTEBC intake: $69 \%$ among those with a non/low intake (approx. 0 servings/day based on a $30 \mathrm{~g}$ serving) compared to $26 \%$ among those with a high intake (approx. 1.9 servings/day). These findings show that the consumption of 'meat \& meat products' is not associated with EGRac status in Irish adults. However, the increased consumption of milks and RTEBC are associated with an improved EGRac status. As RTEBCs are commonly consumed with milk, it is likely that the combined food pattern will be important in determining EGRac status and these findings may be useful in the development of dietary guidelines to improve riboflavin status in the Irish population.

Table 1. Mean intake of milks, meat \& meat products and RTEBC in non/low, medium and high consumers and the prevalence ( $\%$ ) of EGRAC $>1.3$

\begin{tabular}{|c|c|c|c|c|c|c|c|}
\hline & $n$ & Non/Low & $n$ & Medium & $n$ & High & $\overline{\mathrm{p} \text {-value }}$ \\
\hline Milks (g/d) & 373 & 51.4 & 377 & 177 & 376 & 413 & \\
\hline$\%$ EGRAC $>1.3$ & 264 & 70.8 & 231 & 61.2 & 190 & 50.5 & 0.000 \\
\hline Meat \& meat products $(\mathrm{g} / \mathrm{d})$ & 373 & 93.2 & 379 & 174 & 374 & 284 & \\
\hline$\%$ EGRAC > 1.3 & 212 & 56.8 & 231 & 60.9 & 242 & 64.7 & 0.088 \\
\hline RTEBC (g/d) & 451 & 0.1 & 303 & 18.7 & 372 & 56.7 & \\
\hline$\%$ EGRAC $>1.3$ & 310 & 68.7 & 179 & 59.1 & 196 & 52.7 & 0.000 \\
\hline
\end{tabular}

This publication has emanated from research supported in part by a Grant from Science Foundation Ireland (Grant number 16/ERA-HDHL/3357) within the programme of ERA-HDHL - Biomarkers for Nutrition \& Health. (www.healthydietforhealthylife.eu/index.php/64-open-calls/311-derive) The National Adult Nutrition Survey was funded by the Irish Department of Agriculture, Fisheries \& Food under the Food for Health Research Initiative (2007-2012).

1. Kehoe L, Walton J, Hopkins SM et al. (2017) Proc Nutr Soc 78 (OCE3):E

2. Irish University Nutrition Alliance (2012) The National Adult Nutriton Survey www.iuna.net

3. McAuley E, McNulty H, Hughes C et al. (2016) Proc Nutr Soc 75 (3): 405-414. 